\title{
THE DEVELOPMENT OF THE DUCTED FAN NOISE PROPAGATION AND RADIATION CODE CDUCT-LARC
}

\author{
Douglas M. Nark, F. Farassat ${ }^{\dagger}$ D. Stuart Pope ${ }^{\ddagger}$ and Veer Vatsa ${ }^{\S}$ \\ NASA Langley Research Center, Hampton, Virginia
}

\begin{abstract}
The development of the ducted fan noise propagation and radiation code CDUCT-LaRC at NASA Langley Research Center is described. This code calculates the propagation and radiation of given acoustic modes ahead of the fan face or aft of the exhaust guide vanes in the inlet or exhaust ducts, respectively. This paper gives a description of the modules comprising CDUCT-LaRC. The grid generation module provides automatic creation of numerical grids for complex (non-axisymmetric) geometries that include single or multiple pylons. Files for performing automatic inviscid mean flow calculations are also generated within this module. The duct propagation is based on the parabolic approximation theory of R. P. Dougherty. This theory allows the handling of complex internal geometries and the ability to study the effect of non-uniform (i.e. circumferentially and axially segmented) liners. Finally, the duct radiation module is based on the Ffowcs Williams-Hawkings (FW-H) equation with a penetrable data surface. Refraction of sound through the shear layer between the external flow and bypass duct flow is included. Results for benchmark annular ducts, as well as other geometries with pylons, are presented and compared with available analytical data.
\end{abstract}

\section{Introduction}

The prediction of aircraft engine noise, a primary source of sound from aircraft, is important for addressing the issues of community noise and cabin noise control. One of the main aims of Computational Aeroacoustics (CAA) is the development of physics based methodologies for engine noise prediction. This paper describes the development of the ducted fan noise propagation and radiation code CDUCT-LaRC at NASA Langley Research Center. This code calculates the propagation of a given acoustic source ahead of the fan face or aft of the exhaust guide vanes in the inlet or exhaust ducts, respectively. Subsequent to the propagation calculations, the code has the capability of computing the noise radiation field outside the duct. In addition to the ducts being acoustically lined in specified areas, there are other issues that make the propagation and radiation analysis complex:

1. The duct may have a non-uniform circular or annular section. As a result, the flow in the duct may be nonuniform.

2. There may be a pylon in the exhaust duct resulting in one or two C-shaped regions. This will completely change the character of acoustic wave propagation from that of an annular duct and conventional wave propagation theory will then be insufficient.

\footnotetext{
${ }^{*}$ Research Scientist, Structural Acoustics Branch, Aerodynamics, Aerothermodynamics, and Acoustics Competency, AIAA Member

$\dagger$ Senior Research Scientist, Aeroacoustics Branch, Aerodynamics, Aerothermodynamics, and Acoustics Competency, AIAA Associate Fellow

¥Analytical Services and Materials

${ }^{\S}$ Senior Research Scientist, Computational Modeling and Simulation Branch, Aerodynamics, Aerothermodynamics, and Acoustics Competency
}

3. The acoustic lining on the duct wall may be nonuniform (i.e. circumferentially and radially segmented).

CDUCT-LaRC aims to provide a user-friendly environment in which to study these and other issues in the pursuit of low-noise engine designs. In light of this, the development of the code has followed an approach which attempts to separate the main functional capabilities into specific modules. In this way, as improvements or new approaches become available, they may be incorporated more efficiently and without major modification to the user interface. The discussion to follow begins with a description of the major modules within CDUCT-LaRC. This is followed by the presentation of various benchmark cases to provide some confidence in the capabilities of the code. Preliminary results for more complex geometries are then shown to illustrate some of the areas in which the code may be applied. Finally, areas of further development and applicability are discussed.

\section{Modules of CDUCT-LaRC}

CDUCT-LaRC is composed of five distinct modules: input and output specification, Computational Fluid Dynamics (CFD) and acoustic grid generation, background flow calculation, duct acoustic propagation, and duct acoustic radiation. A graphical user interface (GUI) is used for input and output specification and to guide the user through various options offered within the modules. Typically, a session begins with the specification or generation of a background grid.

\section{Grid Generation Module}

The most general noise computation, for either an inlet or aft-fan case, involves the calculation of a background flow (i.e. a CFD calculation). This requires a numerical 
grid about a possibly complex geometry, the generation of which may be outside the interests of many users. In an effort to ease this burden, CDUCT-LaRC offers a grid generation module. Although a complete description of the grid generation techniques is beyond the scope of this paper, a brief discussion of this module is in order. Following guidelines for grid topology, the user simply provides the duct surface geometry in PLOT3D surface format. The input geometry is typically the engine cowl and hub, although a by-pass duct might also include one or two pylons. Based on the defining topology, a background flow grid is automatically generated that is constrained to the input surfaces and clustered in regions of higher curvature. The user has control of grid density, but a complete knowledge of grid generation is not required. It should be noted that it is not necessary to use the grid generation module to generate a background flow grid. It is possible for a user to generate and import a CFD grid, in the appropriate PLOT3D format, if desired.

In addition to the background flow grid, an acoustic grid is used in the propagation calculations. This second grid is necessary because of the conflicting requirements of the background flow and acoustic solution methodologies. While the background flow grid will necessarily include some grid point clustering, this is not desirable for the propagation calculations. On the contrary, as described subsequently, the propagation calculation benefits from numerical grids that are as nearly orthogonal and as uniform as possible. Within CDUCT-LaRC, this acoustic grid is generated directly from the previously created (or imported) background flow grid and constrained to the input surfaces. Using volume Non-Uniform Rational B-Splines (NURBS) techniques, the background grid is splined and the grid points are redistributed uniformly to produce a grid that is more appropriate for the propagation calculations. As was the case with the background flow grid, it is also possible for a user to import an acoustic grid if desired. With the required grids in hand, the background flow and propagation calculations may be performed.

\section{Background Flow and Propagation Modules}

The background flow module provides the ability to study the effects of non-uniform background flow for single or multi-block geometries. Examples include engine inlets and by-pass ducts containing one or two finite-thickness pylons. A well tested and robust flow solver is currently used to produce inviscid solutions, although the ability to calculate viscous flow fields does exist. The required input files are generated within CDUCT-LaRC using supplied ambient and duct flow conditions. For situations in which it is appropriate, the background flow calculation may be avoided and zero or uniform background flow specified. As was the case with the background flow grid, a previously available flow solution may be imported. Regardless of the method in which it is obtained, the background flow solution is then interpolated onto the acoustic grid, again using volume NURBS techniques. The Mach number distribution on the acoustic grid and the input information may then be obtained for the propagation calculations.

The duct propagation module is based on the Boeing CDUCT code developed by Dougherty ${ }^{1}$ and revised by Lan. $^{2}$ This utilizes the parabolic approximation and is an appropriate initial model in that it can satisfy the three requirements listed in the introduction. In CDUCT-LaRC, the code has been extended to allow a user to automatically perform multi-block propagation calculations. Within this module, the grid connectivity is determined and data is transferred from upstream to downstream blocks without user intervention. Because of the approximations in the mathematical model, an orthogonal grid is required for the acoustic calculation. However, many geometries will require isolated regions in which complete orthogonality is not possible. It is believed that such small areas of non-orthogonality will not greatly affect solution quality. Results of the propagation module include the acoustic potential or pressure within the duct. If desired, these results may be passed to the radiation module for acoustic radiation calculations.

\section{Radiation Module}

The duct radiation model is based on the Ffowcs Williams-Hawkings (FW-H) equation with a penetrable data surface. $^{3}$ Testing of this equation for many acoustic problems has shown it to generally provide better results than the Kirchhoff formula for moving surfaces. Based on the background flow conditions and propagation solution, this module calculates the radiated acoustic pressure at various observer locations. These observer locations are specified in the form of a PLOT3D grid file. Currently, the data surface is taken to be the inlet or exhaust plane for inlet or aft-fan cases, respectively. While this provides excellent results in many situations, these choices of data surface location lead to a few limitations. The first appears when large aft or forward radiation angles are of interest for inlet or aft-fan cases, respectively. In these situations, the effects of the engine cowl are not taken into account and the results are essentially symmetric about the data surface. The second issue involves the effects of the shear layer on acoustic radiation.

The shear layer between the bypass flow and external stream can refract the sound waves radiated to the farfield. Radiation results can be improved by including this effect, as well as the reflection of the sound in the bypass region from the solid surface external to the bypass duct surrounding the core flow (see figure 1). One way to achieve this is to extend the bypass duct acoustic calculation, which is based on the parabolic approximation, beyond the exhaust plane and to apply a boundary condition (BC) similar to a 


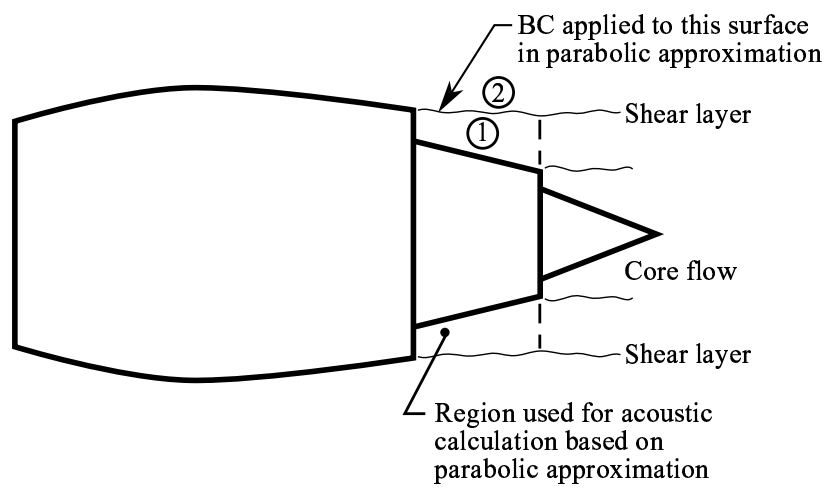

Figure 1 Boundary Regions for the Aft-Fan Geometry

liner BC on the shear layer. Although a great deal of formalism has been avoided up to this point and the $\mathrm{BC}$ is not yet implemented, the novelty of this approach makes it appropriate to present the boundary condition derivation.

On the shear layer, satisfy the following two conditions:

1. The particle displacement must be continuous across the shear layer.

2. The acoustic pressure must be continuous across the shear layer.

The first result in the derivation involves the acoustic velocity normal to the shear layer. This follows a derivation of the liner BC by Myers ${ }^{4}$ as presented by Farassat and Dunn. ${ }^{5}$ The subscripts 0 and 1 are used for the background and acoustic quantities. The particle displacement amplitude normal to the shear layer is also denoted by $q_{3}$ and the time dependence is harmonic with the factor $e^{-i \omega t}$. Following the notation of Farassat and $\operatorname{Dunn}^{5}$ [eq. (3) with $\varepsilon=1$ ], one may write

$$
\begin{aligned}
\frac{\partial q_{3}}{\partial t}=-i \omega q_{3} & =\left[q_{3} \overrightarrow{n_{0}} \cdot\left(\overrightarrow{n_{0}} \cdot \nabla \overrightarrow{u_{0}}\right)+\overrightarrow{u_{1}} \cdot \overrightarrow{n_{0}}\right]^{1} \\
& =\left[q_{3} \overrightarrow{n_{0}} \cdot\left(\overrightarrow{n_{0}} \cdot \nabla \overrightarrow{u_{0}}\right)+\overrightarrow{u_{1}} \cdot \overrightarrow{n_{0}}\right]^{2}
\end{aligned}
$$

where the superscripts 1 and 2 designate regions 1 and 2 in figure $1, \vec{u}$ is the fluid velocity, and $\vec{n}$ is the unit normal to the shear layer.

From equation 1 , one finds

$$
q_{3}=\frac{-u_{1 n}^{1}}{i \omega+\left[\overrightarrow{n_{0}} \cdot\left(\overrightarrow{n_{0}} \cdot \nabla \overrightarrow{u_{0}}\right)\right]^{1}}=\frac{-u_{1 n}^{2}}{i \omega+\left[\overrightarrow{n_{0}} \cdot\left(\overrightarrow{n_{0}} \cdot \nabla \overrightarrow{u_{0}}\right)\right]^{2}}
$$

and

$$
u_{1 n}^{2}=\frac{i \omega+\left[\overrightarrow{n_{0}} \cdot\left(\overrightarrow{n_{0}} \cdot \nabla \overrightarrow{u_{0}}\right)\right]^{2}}{i \omega+\left[\overrightarrow{n_{0}} \cdot\left(\overrightarrow{n_{0}} \cdot \nabla \overrightarrow{u_{0}}\right)\right]^{1}} u_{1 n}^{1}
$$

This result shows that if $u_{1 n}^{1}$ is found on the shear layer from the parabolic approximation method in region 1 , then $u_{1 n}^{2}$ on the external side of the shear layer may be calculated.

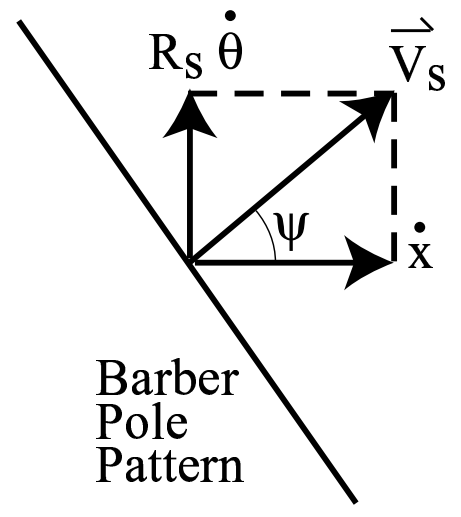

Figure 2 Trace Velocity Description

Next, the derivation of the impedance, $z_{2}$, on the side of the shear layer adjacent to region 2 is presented beginning with the model and assumptions. ${ }^{*}$ First, the shear layer is taken to be infinitely thin, an assumption previously utilized in equation 3. Second, the barber pole pattern of the modes present in the flow region is assumed to persist in the flow region inside the shear layer. This barber pole pattern has a trace velocity on the shear layer surface in the direction normal to itself. Figure 2 shows this trace velocity, $\vec{V}_{s}$, and the method in which it is calculated. This figure also shows a local tangent plane to the shear layer surface which is cylindrical. Assuming the acoustic pressure in a mode to be proportional to $\exp \left[-i\left(\omega t-m \theta-k_{x} x\right)\right]$ where $m$ is the circumferential mode number, $x$ is the axial distance, and $k_{x}$ is the axial wave number, it follows that

$$
\begin{gathered}
\dot{\theta}=\frac{\omega}{m}, \dot{x}=\frac{\omega}{k_{x}} \\
R_{s} \dot{\theta}=\frac{\omega / c_{1}}{m} R_{s} c_{1}=\frac{k R_{s}}{m} c_{1} \\
\dot{x}=\frac{\omega / c_{1}}{k_{x}} c_{1}=\frac{k}{k_{x}} c_{1} \\
V_{s}=\left\|\vec{V}_{s}\right\|=k c_{1} \sqrt{\left(\frac{R_{s}}{m}\right)^{2}+\frac{1}{k_{x}^{2}}} .
\end{gathered}
$$

Here, $R_{S}$ is the local radius of the shear layer (assumed almost constant), $k=\omega / c_{1}$, and $c_{1}$ is the local speed of sound in region 1 of the shear layer.

From Morse and and Ingard ${ }^{6}$ (equation 11.1.21), the impedance, $z_{2}$, is

$$
z_{2}=\frac{\rho_{2} c_{2}}{\left(1+M_{2} \cos \phi\right) \sin \phi}
$$

${ }^{*}$ Note that until the end of this section, we work with the zeroth order quantities (background flow, time independent quantities). For simplicity of notation, we denote regions 1 and 2 by subscripts. 


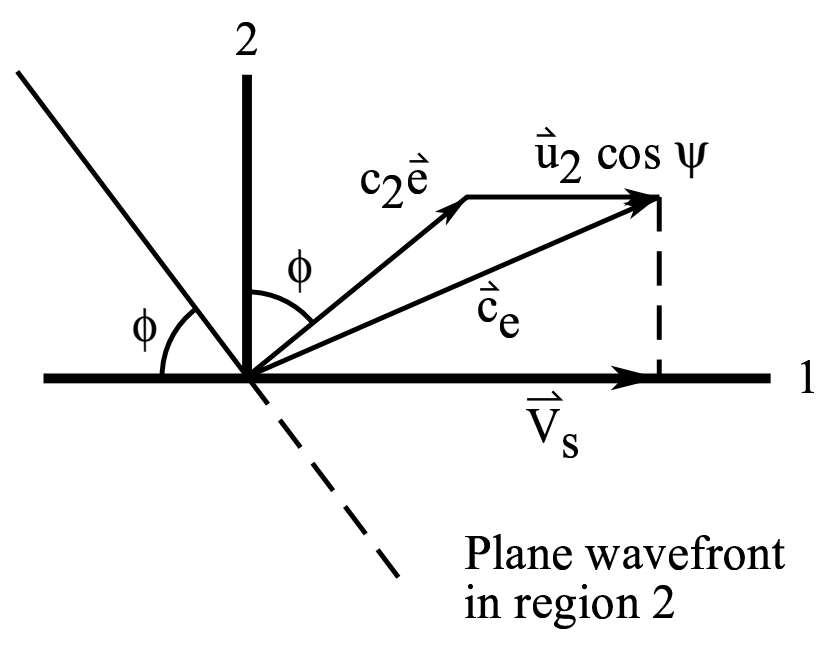

Figure 3 Effective Wave Speed Description

where $M_{2}=u_{2} / c_{2}$ is the Mach number based on the background flow in region 2 and $\phi$ is the preferred direction of propagation of plane waves in region 2. This angle is found purely by kinematic considerations. Figure 3 shows the geometry in the local plane containing $\vec{V}_{s}$ and the local normal to the shear layer which is along axis 2 . Let $\vec{e}=(\sin \phi, \cos \phi)$ be the direction normal to the wavefront. The effective wave speed in region $2, \overrightarrow{c_{e}}$, is

$$
\overrightarrow{c_{e}}=c_{2} \vec{e}+\overrightarrow{u_{2}} \cos \psi
$$

where $\cos \psi=\dot{x} / V_{s}$ is shown in figure 3 . The projection of $\overrightarrow{c_{e}}$ on the shear layer is equal to the trace speed $V_{s}$. This gives

$$
V_{s}=u_{2} \cos \psi+c_{2} \sin \phi
$$

from which it is seen that

$$
\sin \phi=\frac{V_{s}-u_{2} \cos \psi}{c_{2}} .
$$

Using this in equation 8 leads to

$$
z_{2}=\frac{\rho_{2} c_{2}\left(V_{s}-u_{2}\right)}{V_{s} \sqrt{\left(V_{s}-u_{2}\right)^{2}-c_{2}^{2}}} .
$$

The final step in the formulation is that

$$
z_{1}=\frac{u_{1 n}^{2}}{u_{1 n}^{1}} z_{2}
$$

and equation 3 provides an expression for $u_{1 n}^{2} / u_{2 n}^{1}$. It should again be noted that the radiation results to follow use the duct exhaust plane as the data surface and do not utilize this boundary condition; however, implementation is underway.

\section{Benchmark Results}

In order to verify that the various modules function properly, a benchmark noise propagation and radiation study has been performed using a straight annular duct with mean flow. Although the applicability and accuracy of the current propagation module has been documented, ${ }^{2}$ further confidence in its multi-block implementation is desirable. Initial verification of data transfer and retarded time calculations within the radiation module is also of interest. The benchmark geometry is a straight annular duct with dimensions that approximate the more realistic bypass duct presented in sections to follow. As such, the inner radius is $0.285 \mathrm{~m}$ (11.2 in), the outer radius is $0.412 \mathrm{~m} \mathrm{(16.22} \mathrm{in),} \mathrm{and} \mathrm{the}$ overall length is $1.07 \mathrm{~m}$ (42.9 in). The mean flow within the duct was taken to be uniform in the direction of the duct axis at $M=0.4$. The specification of uniform mean flow meant that a background flow grid and calculation were not necessary. Instead, it was possible to simply specify the uniform flow conditions on the acoustic grid. As a side note, this illustrates some of the flexibility of CDUCTLaRC in that propagation calculations are carried out with a previously obtained mean flow solution.

\section{Propagation Calculations}

The aforementioned geometry and mean flow provide an opportunity to perform in-duct propagation calculations and formulate analytical solutions for comparison. The process begins with results for modal propagation in the direction of the mean flow (i.e. positive traveling waves). Assuming the duct axis to run in the $x$-direction from $x=0$ to $x=1.07 \mathrm{~m}$, this implies modal source specification at $x=0$ and approximates the situation of an aft-fan case. The source frequency was taken to be $5000 \mathrm{~Hz}$ and the $(10,1)$ hardwall annular duct mode (corresponding to a cut-off ratio of 3.59) was specified. Figure 4 shows contours of the real part of the complex acoustic potential for this mode on a grid of dimension $17 \times 113 \times 153$ (radial, circumferential, and axial dimensions, respectively). This result is compared with an analytic solution obtained from duct propagation theory in figures 5 and 6 . The plotted data is extracted from grid lines at $\theta=0, r=0.308 \mathrm{~m}$ (12.1 in.) and $r=0.377 \mathrm{~m}$ (14.8 in). As these plots show, the solutions match very well. In order to provide confidence in the data transfer between blocks for multi-block propagation cases, this problem was also run with the duct split into three separate blocks. The result was then compared with the single block result presented in figure 4 . Figure 7 provides an example comparison for data extracted along a grid line at $\theta=0, r=0.308 \mathrm{~m}$ showing that the single and multi-block solutions match.

In addition to the previous cases, an upstream propagation case was also examined. Assuming the duct axis to again run in the $x$-direction from $x=0$ to $x=1.07 \mathrm{~m}$, upstream propagation implies modal source specification 


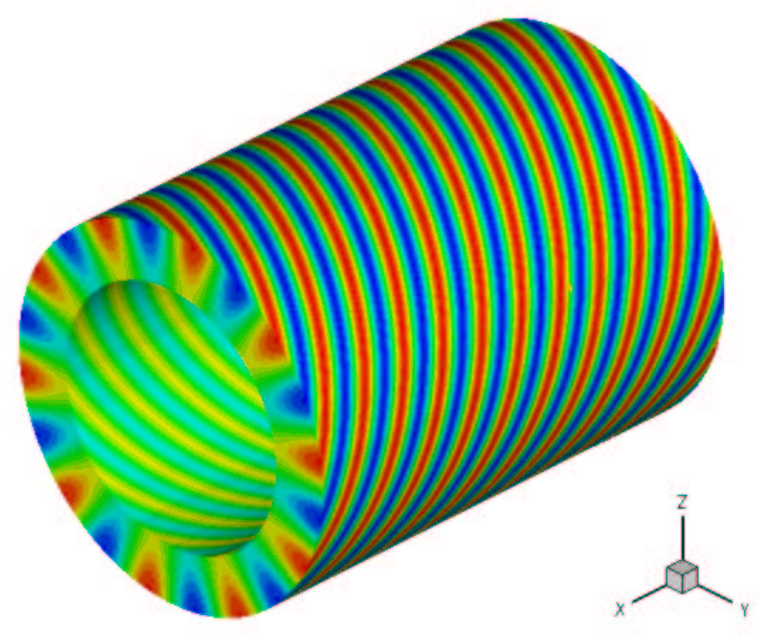

Figure 4 Real Part of the Acoustic Potential - Downstream Propagation $[M=0.4,5000 \mathrm{~Hz},(10,1)$ mode $]$.

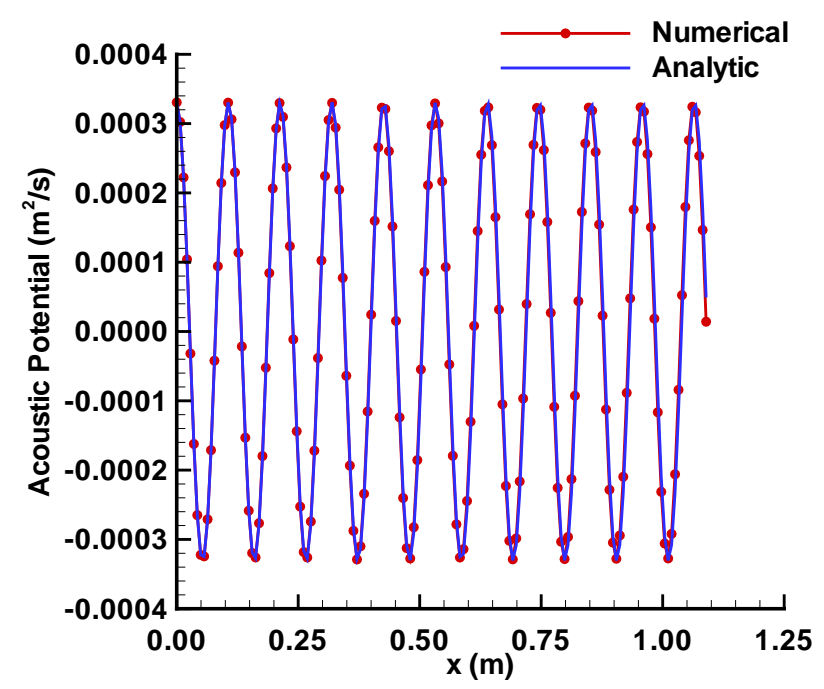

Figure 5 Real Part of Acoustic Potential - Downstream Propagation $[r=0.308 \mathrm{~m}, \theta=0, M=0.4,5000 \mathrm{~Hz},(10,1)$ mode $]$.

at $x=1.07 \mathrm{~m}$ and approximates the situation of an inlet case. The source frequency was again taken to be $5000 \mathrm{~Hz}$ and the $(10,1)$ hardwall annular duct mode was specified. Figures 8 and 9 present comparisons of the CDUCT-LaRC results and the analytic solution from duct propagation theory. The plotted data is extracted from grid lines at $\theta=0, r$ $=0.308 \mathrm{~m}$ (12.1 in.) and $r=0.377 \mathrm{~m}$ (14.8 in). As was the case with the downstream propagation results, the solutions match very well.

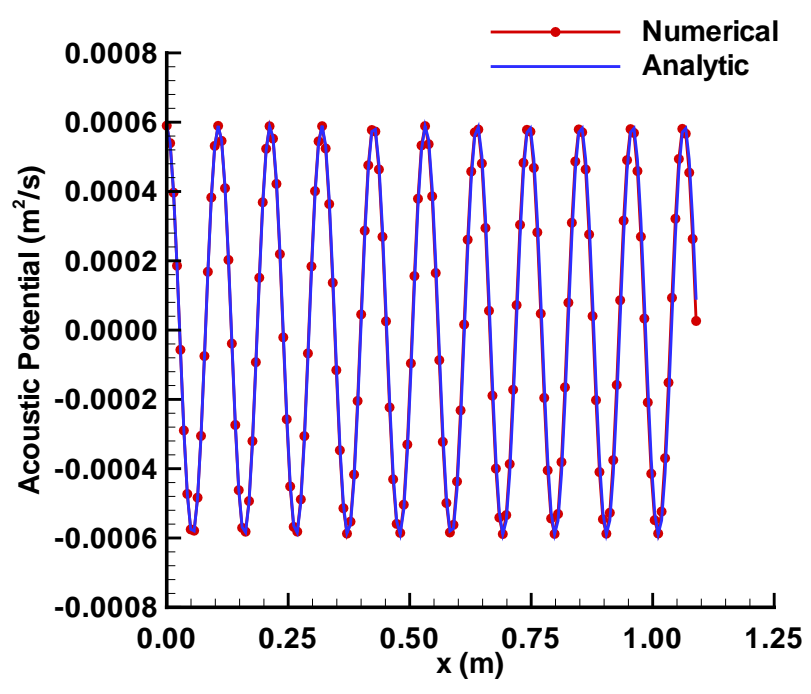

Figure 6 Real Part of Acoustic Potential - Downstream Propagation $[r=0.377 \mathrm{~m}, \theta=0, M=0.4,5000 \mathrm{~Hz},(10,1)$ mode $]$.

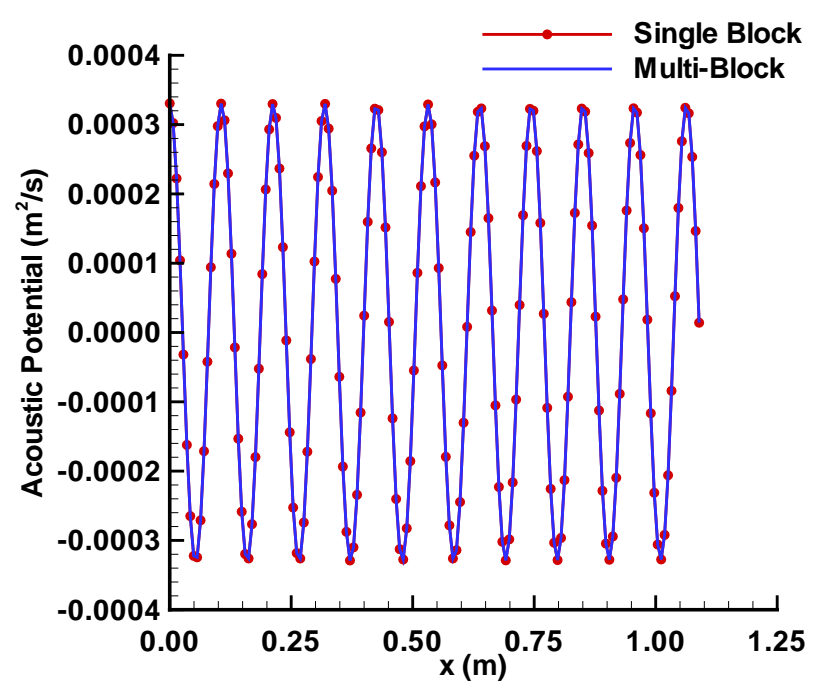

Figure 7 Single and Multi-block Results for the Real Part of the Acoustic Potential $[r=\mathbf{0 . 3 0 8} \mathbf{~ m}, \theta=0, M=0.4, \mathbf{5 0 0 0} \mathbf{~ H z}$, $(10,1)$ mode]. 


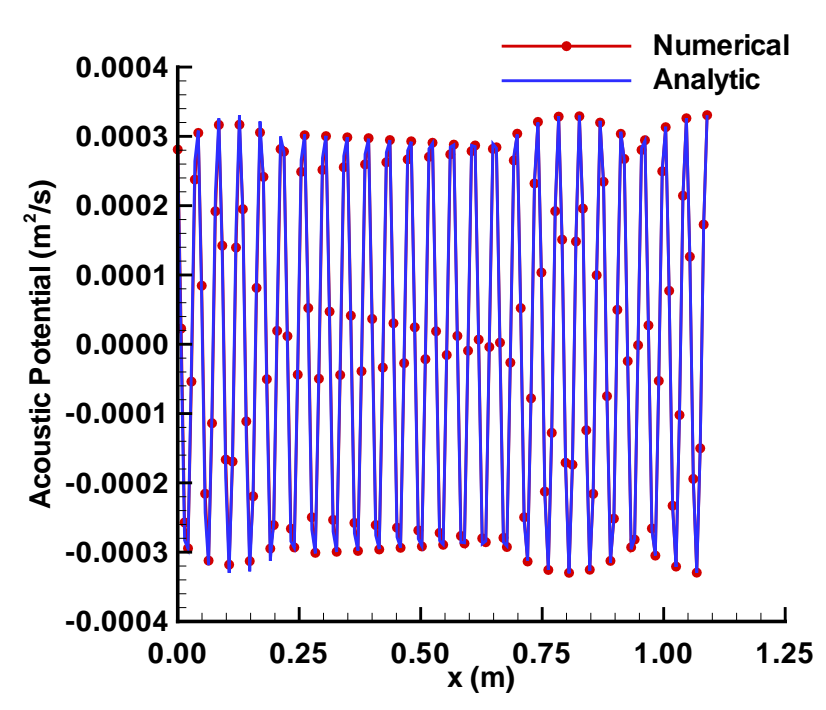

Figure 8 Real Part of Acoustic Potential - Upstream Propagation $[r=0.308 \mathrm{~m}, \theta=0, M=0.4,5000 \mathrm{~Hz},(10,1)$ mode $]$.

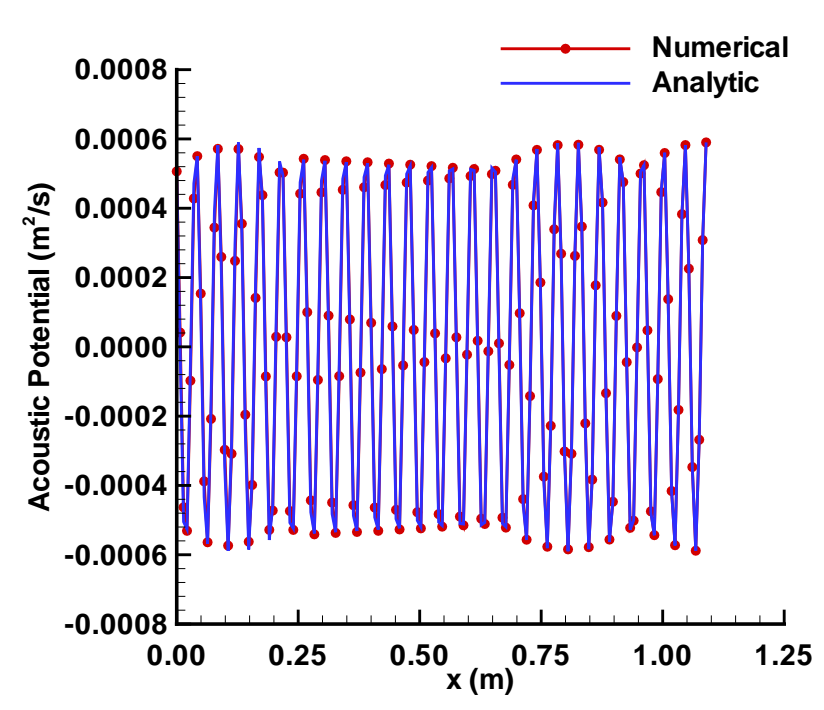

Figure 9 Real Part of Acoustic Potential - Upstream Propagation $[r=0.377 \mathrm{~m}, \theta=0, M=0.4,5000 \mathrm{~Hz},(10,1)$ mode $]$.

\section{Radiation Calculations}

As mentioned previously, CDUCT-LaRC contains a radiation module based on the Ffowcs-Williams Hawkings equation with a penetrable data surface. The benchmark study also entailed using the benchmark aft-fan (downstream) propagation results to calculate the subsequent noise radiation field outside the duct. Radiation calculations were performed on a hemisphere of radius $1.91 \mathrm{~m}$ (75 in) centered on the duct axis in the exhaust plane. Figure 10 shows the radiated pressure ( $\mathrm{dB}$ re: $20 \mu \mathrm{Pa})$ on this hemisphere. Since the benchmark geometry is a straight

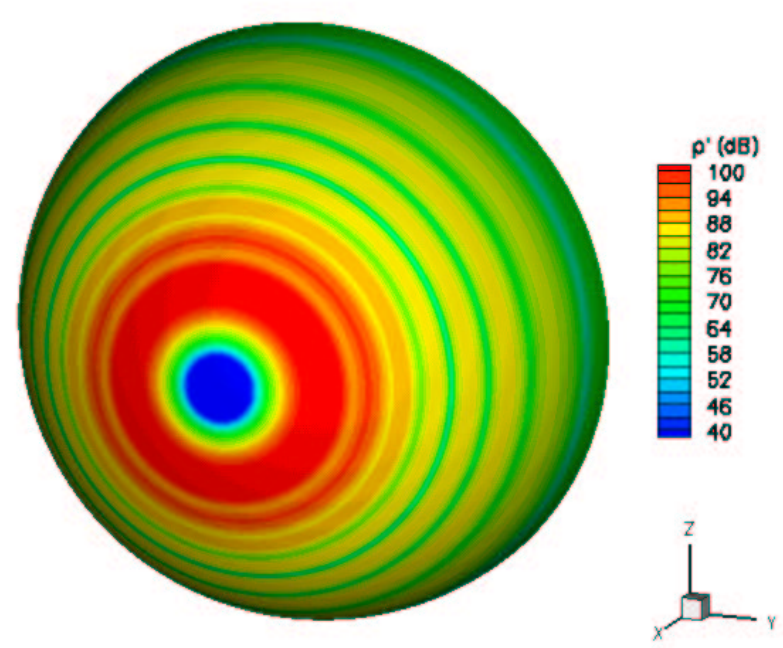

Figure 10 Radiated Sound Pressure Level (dB re: $20 \mu \mathrm{Pa}$ )

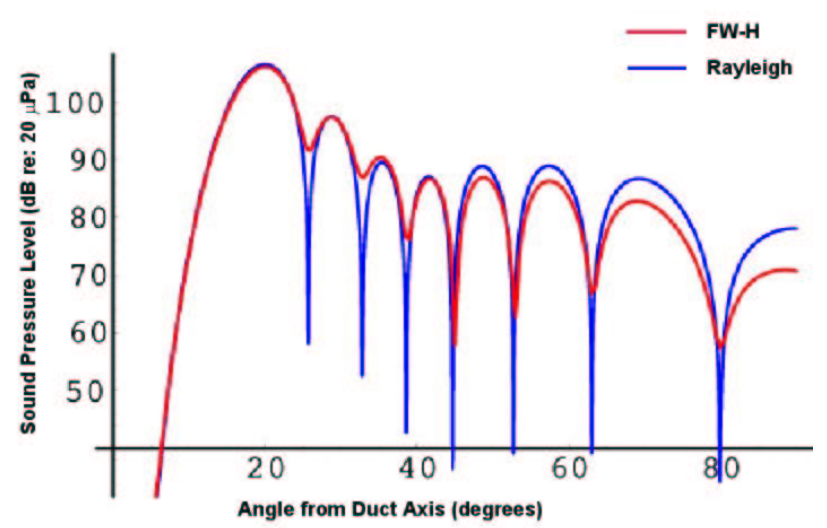

Figure 11 Comparison of CDUCT-LaRC and Rayleigh (Analytical) Radiation Sound Pressure Levels $[5000 \mathrm{~Hz},(10,1)$ mode].

annular duct, a Rayleigh piston in a wall formulation may be used to obtain an analytical result for the exhaust radiation. Figure 11 shows the comparison of the CDUCTLaRC and Rayleigh results along an arc on the observer hemisphere. The solutions compare well as the relative amplitudes and directivity pattern are similar with slight discrepancies in the overall amplitude of some of the minor lobes. In addition to the $(10,1)$ mode, the full series of propagation and radiation calculations were carried out for the $(10,4)$ mode. For the benchmark duct and source frequency of $5000 \mathrm{~Hz}$, this provides a situation in which the propagating wave is much closer to cut-off (cut-off ratio of 1.26). Comparison of the CDUCT-LaRC and Rayleigh results along an arc on the observer hemisphere is presented 


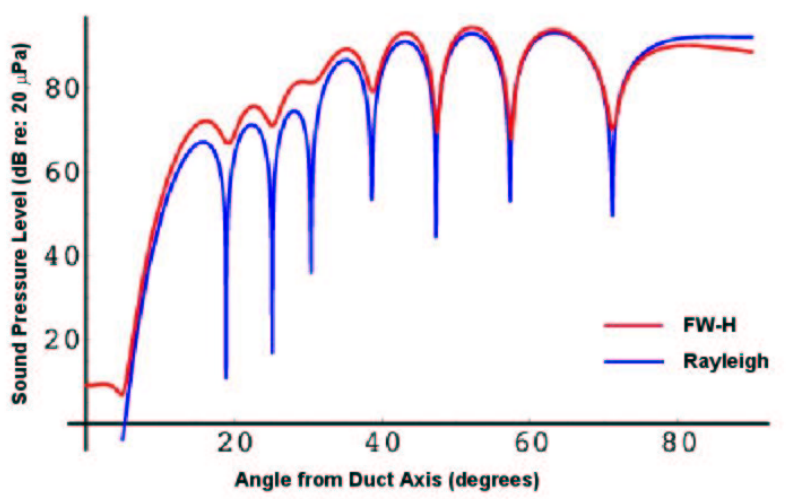

Figure 12 Comparison of CDUCT-LaRC and Rayleigh (Analytical) Radiation Sound Pressure Levels $[5000 \mathrm{~Hz},(10,4)$ mode].

in figure 12 . The relative amplitudes and directivity pattern again compare well and the results of these various benchmark problems provide some justification for performing preliminary calculations on more complex geometries.

\section{Preliminary Results}

In an effort to incrementally work toward more complex geometries, some preliminary calculations were performed on two ducts similar in dimension to the benchmark straight annular duct. The first of these ducts (duct 1) is identical to the benchmark duct except that an infinitely thin pylon is located in the middle third of the duct. The second is a by-pass duct (duct 2) similar to that of a business jet. Both ducts have the same inlet geometry and overall length of $1.07 \mathrm{~m}$ (42.9 in), however, duct 2 has non-constant radial dimensions. Additionally, both ducts have pylons in the same axial location with the pylon of duct 2 being modeled as a NACA 0015 airfoil. Prior to the propagation and radiation calculations, the background flow within the ducts must be established. Since duct 1 is a straight annular duct and inviscid flow is assumed, the background flow was taken to be uniform at Mach number 0.4 (as with the benchmark cases). The background flow module of CDUCT-LaRC was used to obtain the mean flow within duct 2 with an inlet Mach number of 0.4.

With the background flow specified, propagation calculations were performed within the two ducts. Acoustic source specification followed that of the benchmark cases and consisted of specifying the $(10,1)$ hardwall annular duct mode at the duct inlet with a source frequency of $5000 \mathrm{~Hz}$. Figures 13 and 14 show contours of the real part of the acoustic potential for ducts 1 and 2, respectively. In these figures, the fluid flow and modal propagation are in the positive $x$-direction (i.e. the duct exhaust plane is in the foreground). While the typical modal pattern is present, the

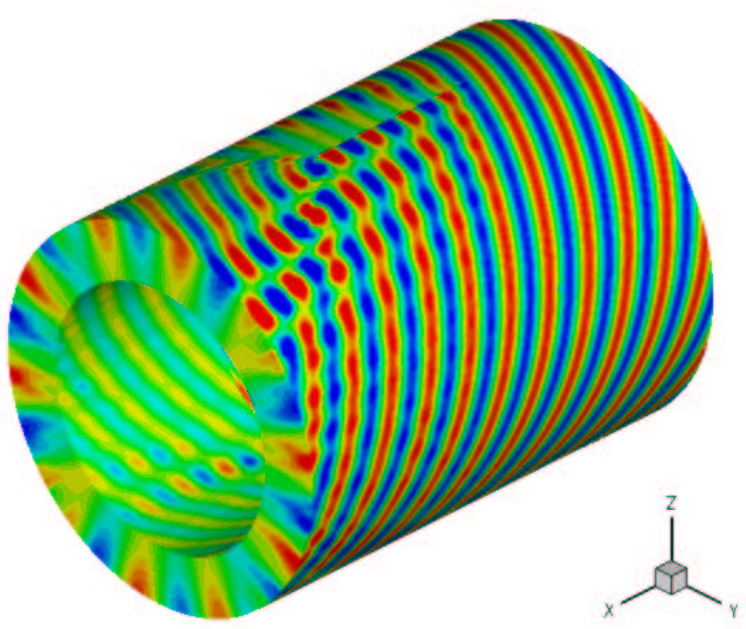

Figure 13 Real Part of the Acoustic Potential - Duct 1 [5000 Hz, (10,1) mode].

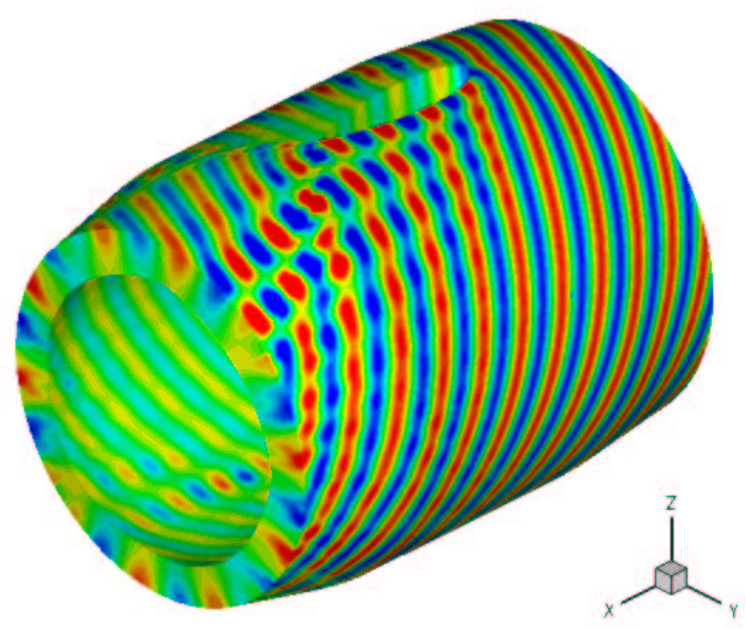

Figure 14 Real Part of the Acoustic Potential - Duct 2 [5000 Hz, (10,1) mode].

effects of the pylon are visible as standing waves are generated within the pylon region. The differences are more evident when the cases are compared with the benchmark annular duct contours shown in figure 4.

The change in modal content and non-uniform mean flow resulting from the pylons may also modify the radiated sound field. As such, the propagation results for ducts 1 and 2 were used to perform farfield radiation calculations. Using the observer grid of the benchmark cases, radiation results were obtained on a hemisphere of radius $1.91 \mathrm{~m}(75 \mathrm{in})$ centered on the duct axis in the exhaust 


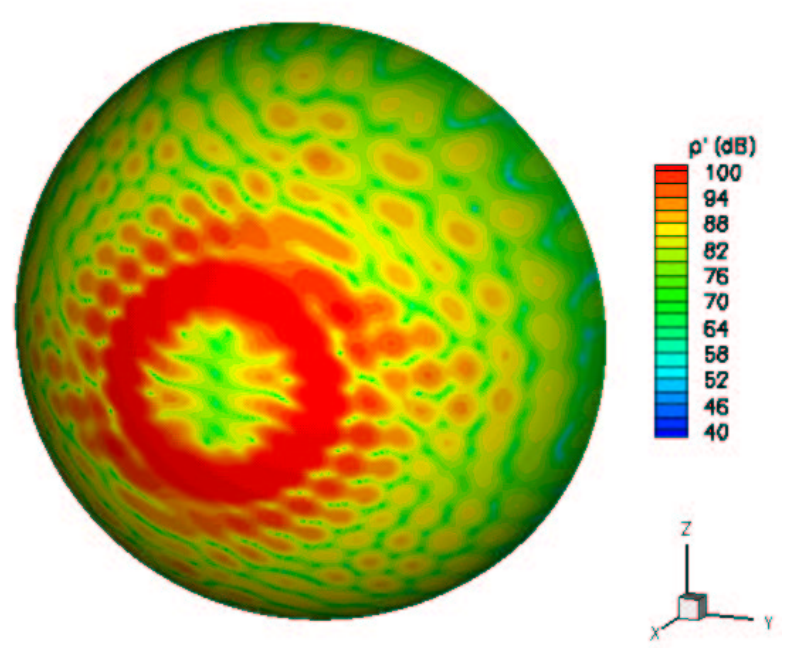

Figure 15 Radiated Sound Pressure Level - Duct 1 (dB re: $20 \mu \mathbf{P a})$

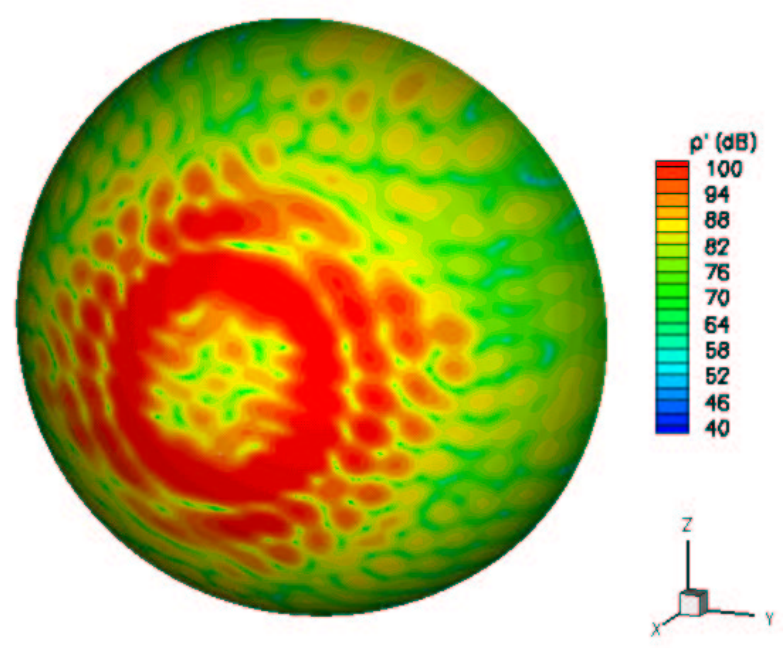

Figure 16 Radiated Sound Pressure Level - Duct 2 (dB re: $20 \mu \mathbf{P a})$

plane. The radiated pressure ( $\mathrm{dB}$ re: $20 \mu \mathrm{Pa})$ on this hemisphere for ducts 1 and 2 are presented in figures 15 and 16, respectively. Comparison of these radiated pressure patterns with the benchmark case (see figure 11) clearly show a modification in the directivity pattern. In light of these results, a focused study of the effects of pylon placement and geometry may prove to be a fruitful area of application for CDUCT-LaRC. Additionally, the ability to model more complex, multi-block geometries may widen the scope of inlet or aft-fan noise calculations that may be investigated.

\section{Concluding Remarks}

The development of CDUCT-LaRC has followed a modular approach with a main goal of providing a physics based acoustic propagation and radiation tool within a user-friendly environment. Compartmentalizing the main functionality provides the ability to make modifications or replace entire modules efficiently and without major changes to the user interface. The current capabilities of the grid generation, background flow calculation, duct acoustic propagation, and acoustic radiation modules which make up CDUCT-LaRC have been described. With the implementation of the shear layer BC formulated herein, larger propagation angles will be attainable and the refraction effects of the shear layer more accurately modeled. It should also be noted that while the benchmark and preliminary results focused on hardwall ducts, the ability to handle radially and circumferentially varying liners exists within the propagation module.

The presented results provide some indication of the capabilities of CDUCT-LaRC, as well as research areas in which the code may be applicable. In particular, the modification of the modal content by the presence of the pylons in the duct, as seen in the results for ducts 1 and 2, is of particular interest. In light of this, implementation of modal decomposition in the post-processing functionality of the code has been initiated and is near completion. Additionally, the ability to handle complex, multi-block geometries within CDUCT-LaRC allows investigation into the effects of design modifications such as radial or circumferential splitters. While there is a great deal of testing to be performed, preliminary results show that the modules for duct propagation and radiation offer appropriate initial models for noise prediction.

\section{References}

\footnotetext{
${ }^{1}$ Dougherty, R. P., "A Wave-Splitting Technique for Nacelle Acoustic Propagation," AIAA Paper 97-1652, 1997.

${ }^{2}$ Lan, J. H., "Turbofan Duct Propagation Model," NASA CR 2001211245, 2001.

${ }^{3}$ Brentner, K. B. and Farassat, F., "Analytical Comparison of the Acoustic Analogy and Kirchhoff Formulation for Moving Surfaces," AIAA Journal, Vol. 36, No. 8, 1998, pp. 1379-1386.

${ }^{4}$ Myers, M. K., "On the Acoustic Boundary Condition in the Presence of Flow," Journal of Sound and Vibration, Vol. 71, No. 3, 1980, pp. 429 434.

${ }^{5}$ Farassat, F. and Dunn, M. H., "A Simple Derivation of the Acoustic Boundary Condition in the Presence of Flow," Journal of Sound and Vibration, Vol. 224, No. 2, 1999, pp. 384-386.

${ }^{6}$ Morse, P. M. and Ingard, K. U., Theoretical Acoustics, McGraw-Hill Book Company, 1968.
} 